\title{
AN ANALYSIS AND A COMPARISON OF INTERCEPTING AND NON-INTERCEPTING BEAM POSITION DETECTORS
}

\author{
Fred Hornstra \\ National Accelerator Laboratory \\ May 1971
}

\section{ABSTRACT}

A fundamental and an important difference exists between beam position detectors with incident beam intercepting electrodes and those without intercepting electrodes. Position detectors with incident beam intercepting electrodes, for example a split secondary emission monitor, are characterized by a highly non-linear transfer function which is also beam size dependent. Quantitative beam position information generally is not available, and detectors of this configuration will present difficulties when used in feedback arrangements to stabilize beam position. On the other hand, detectors with non-intercepting electrodes have a well behaved linear transfer function invariant with beam size and position. The latter detectors are superior in situations requiring quantitative beam position information, good dynamic range and stability. A comparison of signal available per incident beam particle per $\mathrm{g} / \mathrm{cm}^{2}$ of scattering material in the beam also favors a detector with non intercepting electrodes. 


\section{INTRODUCTION}

The transfer functions for two different configurations of beam detectors are analyzed and compared for use in stabilizing beam positions in external beam lines. A feedback arrangement is implied wherein an error signal derived from the position detector is sent to a steering magnet to restore the beam to its desired position. The two different detector configurations are characterized by having either beam intercepting or nonintercepting electrodes. Also presented for typical detectors in both configurations is a normalized quantity representing the signal available per incident beam particle per $\mathrm{g} / \mathrm{cm}^{2}$ of scattering material.

\section{NON-INTERCEPTING DETECTOR}

The detection scheme of a non-intercepting detector (1) is depicted in Fig. 1. Here a beam passes over charge collection electrodes. Ionized electrons (or ions) are collected by a small sweeping electric field normal to the electrodes. Simple electronics yields a transfer function $e_{0}(x)$, which is proportional to beam displacement as indicated. Sensitivity

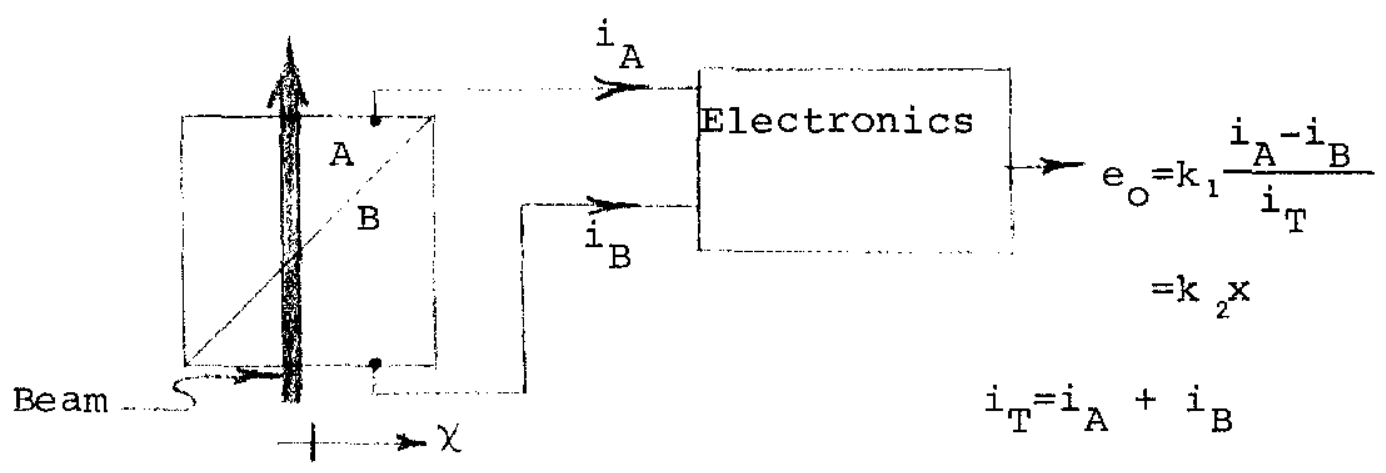

Figure 1. Non-intercepting beam position detector. 
of the detector, defined as $\frac{\mathrm{de}}{\mathrm{dx}}$, is a constant independent of beam position and size. When the beam is centered on the detector $i_{A}=i_{B}=\frac{i_{T}}{2}$ which means that for this condition, the common mode signal is equal to one-half the total signal.

The detector depicted in Fig. 2 enjoys the same transfer function and sensitivity as the one above; however, the common mode signal is greatly reduced so that when the beam is nearly centered, higher sensitivity is in fact achievable.

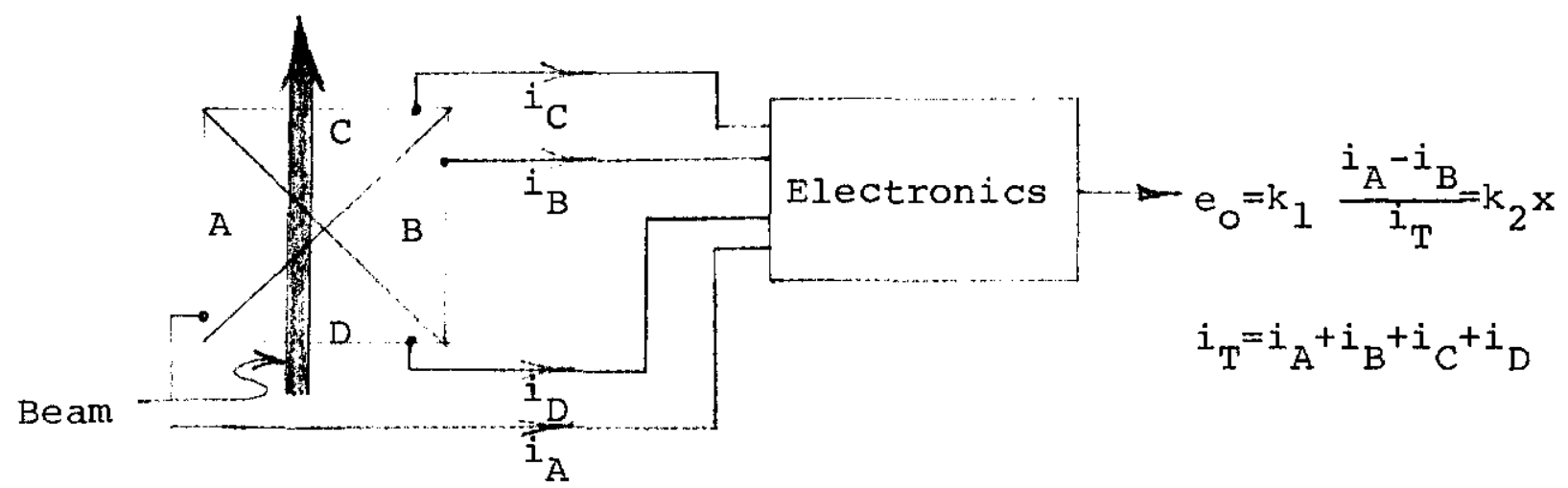

Figure 2. Four segment non-intercepting beam position detector.

\section{INTERCEPTING DETECTOR}

Frequently, split intercepting electrodes such as SEM's, ion chambers, delta-ray emitting foils (insulated targets) etc. are used for sensing beam position as indicated in Fig. 3. The transfer functions of all these devices are related by constants which represent the relative gains of the detectors. The characteristic behavior of these devices is an output and a sensitivity which have strong non-linear dependence on beam size and on beam position as illustrated in the following analysis. 


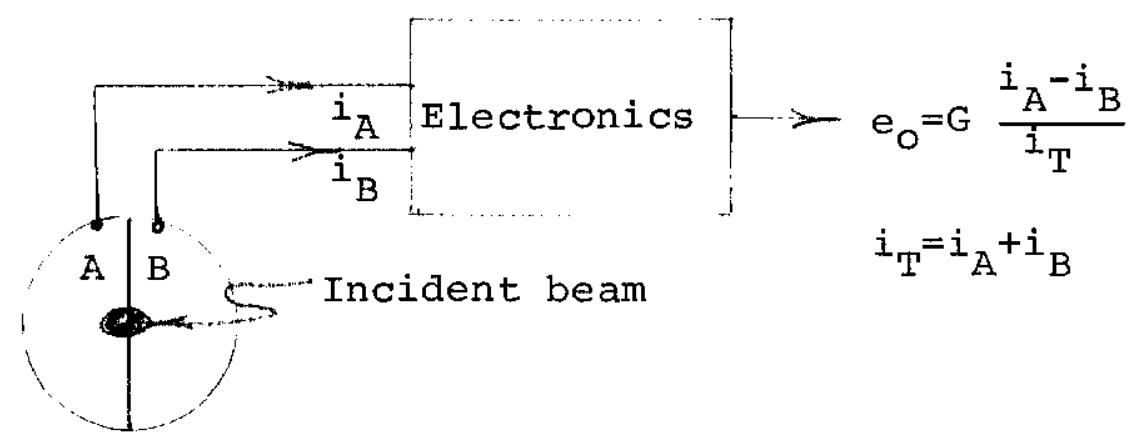

Figure 3. Intercepting beam position detector.

A normal beam, i.e., one which uniformly populates phase space, exhibits a distribution in real space, $x$ and $y$, as follows: (2)

$$
\frac{j}{j_{0}}=\left(1-\frac{x^{2}}{A^{2}}-\frac{y^{2}}{B^{2}}\right)^{2} \begin{aligned}
& -1<\frac{x}{A}<+1 \\
& -1<\frac{\mathrm{Y}}{B}<+1
\end{aligned}
$$

where $\frac{j}{j_{0}}$ is the instantaneous relative current density and $A$ and $B$ are the semiwidths of the beam in the $x$ and $y$ direction respectively.

Since motion in one direction only is considered for a given detector, no loss of generality will result if $y=0$ then,

$$
\frac{j}{j_{0}}=\left(1-\frac{x^{2}}{A^{2}}\right)^{2} \quad-1<x<+1
$$

which is illustrated in Fig. 4. 


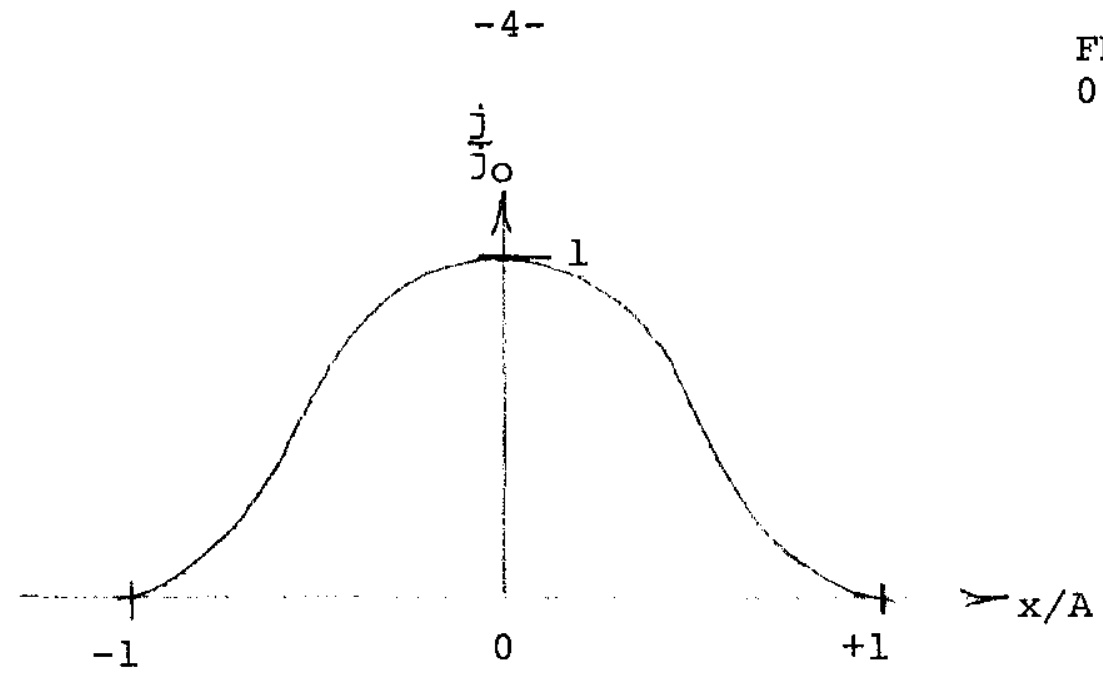

Figure 4. Current density across the beam in

$\mathrm{F}-231$

0880

\section{real space resulting from a uniform density in phase space.}

By necessity

$$
\int_{-1}^{+1} \frac{j}{j_{0}} d\left(\frac{x}{A}\right)=i(t)
$$

the instantaneous current of the beam.

Consider now such a beam incident on a detector with a displacement as illustrated pictorially in Fig. 5 and graphically in Fig. 6.

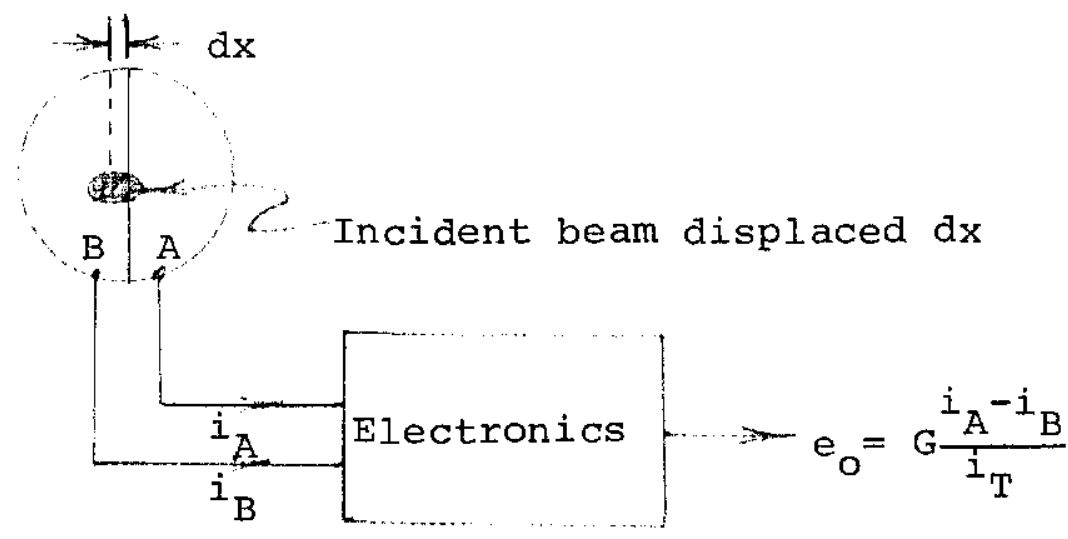

Figure 5. Incident beam displaced on intercepting electrodes. 


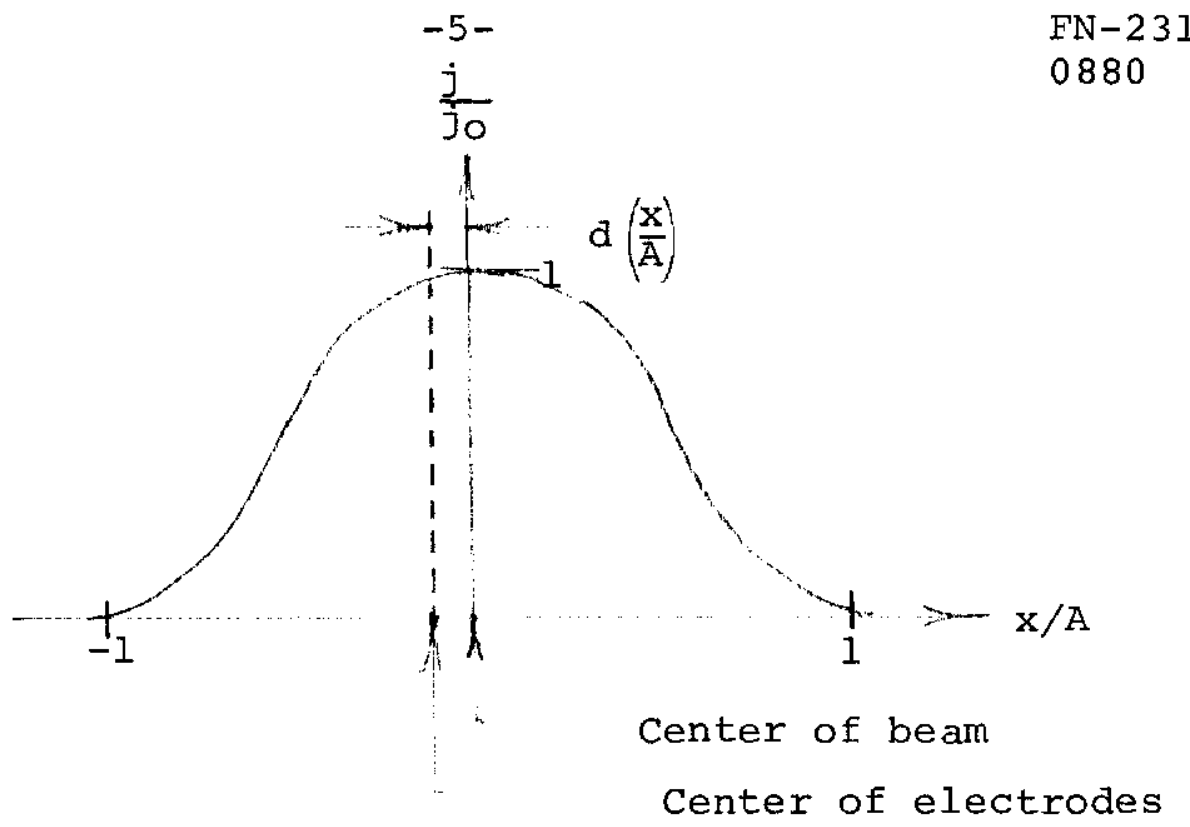

Figure 6. Current density across beam displaced Erom center of electrodes in Figure 5 .

Now $\begin{aligned} i_{A} & =\frac{i_{T}}{2}-\frac{j}{j_{O}} d\left(\frac{x}{\bar{A}}\right) \\ i_{B} & =\frac{i_{T}}{2}+\frac{j}{j_{O}} d\left(\frac{x}{\bar{A}}\right)\end{aligned}$

and $d\left(e_{0}\right)=G \frac{i_{A}{ }^{-i} B}{i_{T}}=2 G \frac{j}{j_{O}} d\left(\frac{x}{A}\right)$

$$
\begin{aligned}
& \text { Sensitivity } \equiv \frac{d e_{0}}{d \frac{x}{A}}=\frac{2 j}{j_{0}}=\frac{2 G}{A^{4}}\left(A^{2}-x^{2}\right)^{2}
\end{aligned}
$$

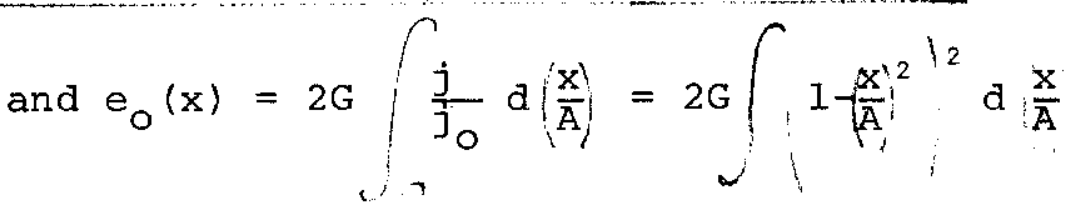

$$
\begin{aligned}
& =2 \mathrm{G} \mid\left[1-2\left(\frac{\mathrm{x}}{\overline{\mathrm{A}}}\right)^{2}+\left(\frac{\mathrm{x}}{\mathrm{A}}\right)^{4} \mathrm{~d}\left(\frac{\mathrm{x}}{\mathrm{A}}\right)\right.
\end{aligned}
$$$$
c^{\prime}
$$

$\operatorname{Transfer}$ Function $\equiv e_{0}(x)=2 G \frac{x^{\prime}}{\frac{A}{3}} \frac{2 G}{\bar{A}^{\prime}}+\frac{2 G}{5} \frac{x^{5}}{A}-1<\frac{x}{\bar{A}}<+1$ 
Several important characteristics are observable from these equations.

1) The output and the sensitivity exhibit a high degree of dependence both on beam position and beam size.

2) The smaller the beam, the higher the sensitivity.

3) The sensitivity is zero for $x>A, i . e$. the output, $e_{0}$, is saturated at $-1>\frac{x}{\mathrm{~A}}>1$.

4) For displacements $\frac{x}{\bar{A}}<1$, the output is linear and the sensitivity is constant at its maximum.

5) The common mode signal is $\frac{i_{T}}{2}$ for beam centered.

The increased sensitivity for small beam widths seems desirable in any situation since, in general, the smaller the beam, the greater the precision with which it must be controlled. The common mode signal could be reduced by making the intercepting detector of four segments; however, the result will be an undesirable coupling of the horizontal and vertical output. The nonlinear transfer function for this detector configuration is undesirable in a feedback arrangement since nonlinearity in the system transfer function can lead to instabilities. The nonlinearity is particularly bothersome if one wishes to bias the beam off-center on the detector to compensate, say, for a misalignment between the detector and a target. The beam size itself may be varying in time, and since the sensitivity varies as the fourth power of the beam size, subtle trouble may be anticipated. Finally, unless the beam is centered, no quantitative position information is available. 


\section{SIGNAL AND INTERACTING MATERIAL}

A normalized gain quantity representing the signal available per incident beam particle per $\mathrm{g} / \mathrm{cm}^{2}$ of interacting material will now be compared for typical detectors in the intercepting and non-intercepting electrode configuration.

Split insulated targets used as intercepting detectors at Brookhaven National Laboratory ${ }^{\left({ }^{3}\right)}$ are $40 \mathrm{mils}$ thick and yield 0.14 delta ray electron per incident particle. The normalized gain quantity for this detector is

$$
v \frac{0.56 \mathrm{e}^{-}}{\text {Incident Particle }\left(\mathrm{g} / \mathrm{cm}^{2}\right)}
$$

Used as split intercepting position detectors, secondary emission foils may be $20.5 \mathrm{mils}$ thick and yield $\sim 0.01$ electrons per incident particle. The normalized gain quantity for this detector is

$$
\frac{3 \mathrm{e}^{-}}{\text {Incident Particle }\left(\mathrm{g} / \mathrm{cm}^{2}\right)}
$$

A gas ion chamber collecting electrons or ions with the non-intercepting electrode configuration has a normalized gain quantity for minimum ionizing particles as follows:

$$
v \frac{20 \text { Electrons }}{\text { Incident Particle }\left(\mathrm{g} / \mathrm{cm}^{2}\right)}
$$


Table I shows the comparison of the above types of detectors.

TABIE I

Comparison of Normalized Gain for Three Typical Beam Position Detectors

\begin{tabular}{l|c|c}
\hline Detector & $\begin{array}{c}\text { Electrode config- } \\
\text { uration }\end{array}$ & $\begin{array}{l}\text { Normalized Gain } \\
\text { electron }\end{array}$ \\
\hline Insulated Target & $\begin{array}{c}\text { Intercepting } \\
\text { Intercepting } \\
\text { SEM }\end{array}$ & 0.4 \\
Ion Chamber & Non-Intercepting & 20 \\
\hline
\end{tabular}

In all fairness one has to point out that the secondary emission monitor may require a vacuum chamber with attendant windows since pressures in beam lines frequently are too high for these monitors. Likewise, for low intensity incident beams, it may be advantageous to have an ionization chamber pressure higher than that permitted in the beam line. As a result, an "anti-vacuum" chamber with attendant windows would be required but for high incident beam intensities ( $\sim$ l ma) the residual gas in the beam lines may provide adequate signal so that no additional intercepting material is presented to the beam. If the incident beam is traveling in air, the ionization device is by far the simplest to implement. In all cases, however, this chamber retains a superior ratio of signal to beam intercepting material. Finally, of all the position detectors, the ion chamber with non-intercepting electrodes is the one in which the signal can be increased most conveniently by simply lengthening the detector. 
A detector with non-intercepting electrodes possesses a well behaved linear transfer function giving quantitative position information invariant of incident beam size and position. If gas ionization is the signal source, such detectors have a superior ratio of signal per $\mathrm{g} / \mathrm{cm}^{2}$ of intercepting material. Increased signal level can be easily achieved by lengthening the detector or increasing the gas pressure.

Detectors with beam intercepting electrodes are characterized by a highly non-linear transfer function which is beam size dependent. In general, quantitative beam position information is unavailable. They have a less favorable ratio of signal per $\mathrm{g} / \mathrm{cm}^{2}$ of intercepting material; furthermore, their signal level per incident beam particle cannot be increased without difficulty. 


\section{REFERENCES}

1 Kirchgesener, J. L. et. al., PPAD64E, Princeton-Pennsylvania Accelerator, Princeton, New Jersey, Apri1 11, 1968.

2 Banford, A. P., "The Transport of Charged Particle Beams" Chapter 2, E. \& F. N. Spon Limited, 1966.

3 Bennett, G. W., "Insulated Target Instrumentation for the AGS Slow External Beam, IEEE Trans Nucl Sci NS-16, June 1969, p. 637 . 